\title{
Tiroidectomía profiláctica en niños y jóvenes con cáncer medular tíroideo hereditario: Experiencia chilena
}

\author{
Claudia Ramos S ${ }^{1}$, Dagoberto 0 jeda $S^{3}$, David Cohn $\mathbf{M}^{3}$, \\ Nelson W ohllk $\mathbf{G}^{1,2}$. \\ Prophylactic thyroidectomy in \\ children and young people with \\ hereditary medullary thyroid \\ carcinoma: A Chilean experience
}

Background: With the availability of the RET proto-oncogene genetic testing, it is possible to perform prophylactic total thyroidectomy among carriers of RET mutation. Aim: To evaluate the histological findings and the effects of the prophylactic total thyroidectomy in firstdegree relatives of Chilean patients with multiple endocrine neoplasia type 2 (MEN 2) based on the Ret proto-oncogen analysis. Subjects and Methods: Nineteen patients belonging to 11 MEN 2 families underwent total thyroidectomy. Of these, 16 either with $\mathrm{C}$ cell hyperplasia $(\mathrm{CCH})$ or microscopic medullary thyroid carcinoma (MTC) were selected for the final analysis. Results: The age at the moment of thyroidectomy ranged from 3 to 24 years (median 9.5). The most common mutation was located in codon 634 (69\%) followed by codon 620 (25\%). Histopathology revealed MTC in 13 patients (81\%, youngest 3 years, oldest ones 19 and 24 years) and $\mathrm{CCH}$ in 3. A significant correlation was observed between basal preoperative serum calcitonin/tumor size ( $r=0.53, \mathrm{P}<0.05)$ and age/tumor size ( $r=0.56, \mathrm{P}<0.03$ ), but not between basal preoperative serum calcitonin and age. Stimulated preoperative calcitonin levels were confounding and not useful for differentiating CCH from MTC. None of patients in whom cervical dissection was done (9/16) presented lymph node metastases, including the oldest ones. All patients but the older ones were biochemically cured after a mean of 5 years of follow-up. Conclusion: Prophylactic total thyroidectomy should be done early in life because there is an age-dependent progression from HCC to MTC. MTC often precedes biochemical detection of the disease (Rev Méd Chile 2005; 133: 1029-36).

(Key Words: Multiple endocrine neoplasia type 2; Thyroidectomy; Thyroid neoplasms)

Recibido el 27 de enero, 2005. Aceptado el 13 de mayo, 2005.

Trabajo financiado parcialmente por Proyecto FONDECYT 1980135.

Presentado al XV Congreso Chileno de Endocrinología y Metabolismo, Arica 13 al 15 de noviembre de 2003. Premio al mejor trabajo libre de becados.

${ }^{1}$ Sección Endocrinología, Hospital del Salvador, Facultad de Medicina Universidad de Chile, División Oriente.

2Instituto de Estudios Médicos Avanzados.

${ }^{3}$ Departamento de Cirugía, Hospital del Salvador.

Correspondencia a: Nelson Wohllk G. Rancagua 835, Provi-

dencia. Fax: 251 8536. E mail: nwohllk@gmail.com 
Tas neoplasias endocrinas múltiples tipo 2 ـ(NEM 2) son síndromes hereditarios autosómicos dominantes de alta penetrancia y de expresión fenotípica variable. Se dividen en 3 subtipos: NEM 2A, NEM 2B y cáncer medular tiroideo familiar. Las glándulas afectadas (tiroides, médula supramenal y paratiroides) se comprometen inicialmente en forma de hiperplasia y a partir de ésta se produce el desarmollo tumoral ${ }^{1}$. En el caso del tiroides, la primera manifestación es la hiperplasia de las células C (HCC), que evoluciona al cáncer medular del tiroides (CMT), componente principal y factor pronóstico determinante de las NEM $2^{2,3}$.

Hace más de 30 años se inició el estudio prospectivo del CMT hereditario, describiéndose la asociación con feocromocitoma ${ }^{4}$. Al mismo tiempo, varios laboratorios desamollaron el radioinmunoanálisis (RIA) para medir calcitonina plasmática ${ }^{5,6}$. La medición de calcitonina estimulada con pentagastrina permitió detectar y tratar precozmente a los pacientes con NEM 2, antes de hacerse sintomáticos ${ }^{7}$.

El estudio genético de las NEM 2 comenzó a principios de la década 1980-89, descubriéndose en 1993 que mutaciones de la línea germinal del proto-oncogen RET eran las causantes de este síndrome, y que unos pocos codones mutados eran responsables de más de $95 \%$ de todos las NEM $2^{8-}$ 12. Esta técnica ha permitido la detección de los portadores de mutaciones del RET en forma mucho más precoz y sensible que con la calcitonina estimulada, ofreciendo la posibilidad de realizar la tiroidectomía profiláctica (TP) aun antes de que se desarrolle el CMT, eliminando los falsos positivos y negativos que se producen con la prueba de estimulación y excluyendo de mayor estudio a los familiares que no son portadores de la mutación ${ }^{13-}$ 15. En el año 2003, el estudio EUROMEN (European Multiple Endocrine Neoplasia) confirmó datos anteriormente publicados respecto a que la progresión temprana del CMT es dependiente de la edad y de la mutación específica del codón (correlación genotipo- fenotipo $)^{16,17}$. Existe también una correlación directa entre el tamaño tumoral y niveles de calcitonina preoperatoria, extensión extratiroidea, compromiso ganglionar cervical y metástasis a distancia ${ }^{18}$. Resulta así indispensable intervenir precozmente a los individuos asintomáticos, portadores de mutaciones de línea germinal del protooncogen RET, con el fin de evitar la progresión tumoral y sus consecuencias.
En nuestro medio, el análisis genético ha intentado abarcar a todos los casos del país con CMT esporádico o hereditario, habiéndose evaluado a la fecha más de 150 individuos, estudio que ha sido informado parcialmente ${ }^{19}$.

Los objetivos de este trabajo fueron evaluar: los hallazgos histológicos, las complicaciones quirúrgicas y los resultados terapéuticos de la TP en familiares asintomáticos de pacientes chilenos con NEM 2, basados en el análisis genético del protooncogen RET.

\section{PACIENTES y MÉTOdo}

Pacientes. A través de la historia clínica se identificaron 22 pacientes que pertenecen a 9 familias con NEM 2A y a 2 con CMT familiar; 105 familiares de primer grado fueron sometidos a extracción de ADN proveniente de leucocitos periféricos y estudio genético del proto-oncogen RET de los exones $10,11,13,14,15$ y 16, identificándose 25 portadores de la mutación. A todos ellos se les aconsejó la TP. Desde 1997 a la fecha se han operado 19 individuos y los 6 restantes no han sido intervenidos quirúrgicamente por diferentes razones: el médico tratante de una niña de 3 años con retardo mental severo y una epilepsia de difícil manejo prefirió posponer la cirugía; un joven de 16 años con una mutación considerada de bajo riesgo de desarrollar CMT precozmente (V804M) no ha sido operado por razones económicas, sin embargo después de 3 años de seguimiento los niveles de calcitonina se han mantenido normales y la ecografía tiroidea no ha mostrado desarrollo de nódulos. En 3 niños de 3, 10 y 12 años, sus padres rechazaron la cirugía y actualmente se desconoce su paradero y finalmente un sujeto de 75 años, sin nódulos ecográficos, rechazó mayor estudio. En este estudio solo se incluyeron sujetos asintomáticos, en cuya biopsia quirúrgica se encontró HCC o CMT $\leq 10 \mathrm{~mm}$ (cáncer microscópico), ya que este tamaño es considerado el adecuado para evaluar el impacto de la cirugía profiláctica ${ }^{16}$. De este modo, 16 sujetos fueron enrolados, a los cuales se les realizó tiroidectomía total como procedimiento estándar. Debido a que no existe consenso respecto a la disección ganglionar, ésta se realizó en todos los mayores de 10 años y en los de $\leq 10$ años en caso de existir ganglios clínicamente sospechosos. 
El estudio genético fue aprobado por el Comité de Ética del Hospital del Salvador. La decisión de realizar TP se tomó en conjunto con los padres de los pacientes y se basó en la experiencia internacional del manejo de portadores de la mutación del RET ${ }^{13-17,20,21 .}$

Método. Previo a la cirugía, se realizó screening para feocromocitoma midiendo metanefrinas y catecolaminas urinarias en $24 \mathrm{~h}$. La calcitonina plasmática se midió entre los años 1997 y 2002 por RIA (Diagnostic Products Corporation, DPC, Los Angeles, USA, valor normal $<50 \mathrm{pg} / \mathrm{ml}$, límite inferior de detección de $16 \mathrm{pg} / \mathrm{ml}$ ) y posteriormente, mediante electroquimioluminiscencia ultrasensible (IMMULTE ${ }^{\circledR}$, valor normal $<8,4 \mathrm{pg} / \mathrm{ml}$, límite inferior de detección de $2 \mathrm{pg} / \mathrm{ml}$ ). Su medición se efectuó en 15/16 pacientes.

Todas las muestras quirúngicas fueron sometidas a examen histopatológico y a estudio inmunohistoquímico para calcitonina.

El seguimiento postoperatorio fue de 5,38 $\pm 2,08$ años (rango 2-8) y se hizo regularmente midiendo la calcitonina ultrasensible basal y estimulada con pentagastrina, de acuerdo a protocolos previamente reportados ${ }^{22,23}$. En todos los pacientes se realizó ecografía cervical y en aquellos con persistencia de calcitonina elevada se efectuó, además, tomografía axial computarizada (TAC) de cuello, mediastino, tórax e hígado, cintigrama óseo ${ }^{99} \mathrm{~m}$ Tecnecio y cintigrama 99mTc-V-DMSA.

Se correlacionó la edad, tamaño tumoralhistología y valores de calcitonina pre y postoperatorios.

Estadística. Se efectuó mediante el coeficiente de correlación de Pearson (r) considerándose significativo un valor $\mathrm{P} \leq 0,05$.

\section{RESULTADOS}

En la Tabla 1 se muestran las características clínico-histológicas de los pacientes, ubicación de la mutación (codón) y los valores preoperatorios de calcitonina plasmática. Las edades al momento

\section{Tabla 1. C aracterísticas clínicas, bioquímicas, hallazgos histológicos y mutaciones encontradas en los portadores de cáncer medular de tiroides hereditario}

\begin{tabular}{|cccccc|}
\hline $\begin{array}{c}\text { Edad a la } \\
\text { cirugía }\end{array}$ & Género & $\begin{array}{c}\text { Número } \\
\text { codón } \\
\text { pg/ml }\end{array}$ & $\begin{array}{c}\text { CT basal } \\
\text { Preop. }\end{array}$ & $\begin{array}{c}\text { T } \\
(\mathrm{mm})\end{array}$ & $\begin{array}{c}\text { Compromiso } \\
\text { ganglionar (N) }\end{array}$ \\
\hline 3 & $\mathrm{~F}$ & 634 & 74 & 6 & N0 \\
6 & $\mathrm{M}$ & 634 & 87 & 1 & NA \\
7 & $\mathrm{M}$ & 634 & 69 & 4 & NA \\
7 & $\mathrm{~F}$ & 620 & $2,9 *$ & $\mathrm{HCC}$ & NA \\
8 & $\mathrm{~F}$ & 634 & 17 & $\mathrm{HCC}$ & N0 \\
8 & $\mathrm{M}$ & 634 & 60 & 2 & NA \\
9 & $\mathrm{M}$ & 634 & 91 & 1 & N0 \\
9 & $\mathrm{~F}$ & 620 & 36 & 3 & NA \\
10 & $\mathrm{M}$ & 634 & $\mathrm{ND}$ & 1 & NA \\
10 & $\mathrm{M}$ & 620 & 15 & 1 & N0 \\
10 & $\mathrm{~F}$ & 634 & 27 & $\mathrm{HCC}$ & N0 \\
11 & $\mathrm{~F}$ & 634 & 96 & 3 & N0 \\
12 & $\mathrm{M}$ & 634 & 48 & 9 & N0 \\
13 & $\mathrm{M}$ & 620 & 105 & 7 & N0 \\
19 & $\mathrm{~F}$ & 634 & 88 & 9 & 7 \\
24 & $\mathrm{~F}$ & 891 & 96 & 1 & \\
\hline
\end{tabular}

T: tamaño tumoral. HCC: hiperplasia de células C. CT: calcitonina. N0: ausencia metástasis ganglionar. ND: no determinado. NA: no analizado. ${ }^{*}$ Calcitonina ultrasensible. Ver valores normales en método. 
de la cirugía fluctuaron entre 3 y 24 años (mediana 9,5); de los 16 pacientes sometidos a TP, 8 eran hombres. Las mutaciones más frecuentes fueron las del exón 11 codón 634 (69\%), exón 10 codón $620(25 \%)$ y exón 15 codón 891 (6\%).

Como se observa también en la Tabla 1, todos los pacientes presentaron algún tipo de alteración histológica: HCC en 3 sujetos y CMT microscópico en 13. La calcitonina basal preoperatoria se encontró levemente elevada en 10/15 pacientes (todos ellos con CMT) y normal en 5 (2 con CMT y 3 HCC); sensibilidad 83\%, especificad 100\%, valor predictivo positivo $100 \%$, valor predictivo negativo $60 \%$. Además se correlacionó directamente con el tamaño tumoral-hallazgo histológico $(\mathrm{r}=0,53 \mathrm{P}<0,05)$, pero no con la edad al momento de la cirugía $(r=0,36 \mathrm{P}=0,18)$. La prueba de calcitonina estimulada con pentagastrina en el preoperatorio sólo se realizó en 7 pacientes (datos no mostrados), ya que la indicación de realizarla en ese período es discutible; los niveles se correlacionaron con la edad $(\mathrm{r}=0,79 \mathrm{P}=0,033)$, pero no con el tamaño tumoral-hallazgo histológico $(r=0,733 \mathrm{P}=0,061)$. En 2 pacientes con HCC, la calcitonina preoperatoria estimulada resultó francamente elevada y en un paciente con CMT de 7 $\mathrm{mm}$, fue normal. Ninguno de los sujetos en los cuales se realizó disección ganglionar cervical (9/ 16) se encontró compromiso metastásico, incluyendo los de 19 y 24 años.

En la Figura 1, se puede observar la correlación entre edad de la tiroidectomía y el tamaño tumoral. El individuo más joven se escapa de la tendencia, no obstante lo cual, se mantiene una correlación directa entre ambos parámetros ( $\mathrm{r}=$ $0,56 \mathrm{P}<0,03)$.

Como se observa en la Figura 2, en el postoperatorio 14/16 pacientes presentaron valores no detectables de calcitonina basal (incluyendo 8 de 10 que tuvieron niveles elevados de calcitonina basal preoperatoria). Los 2 de mayor edad mantuvieron niveles detectables.

La prueba de calcitonina estimulada postoperatoria (datos no mostrados) efectuada en 13/14 sujetos con calcitonina basal no detectable, arrojó también valores bajos, confirmando la cura bioquímica (normalización de calcitonina plasmática) después de un seguimiento de 5 años promedio.

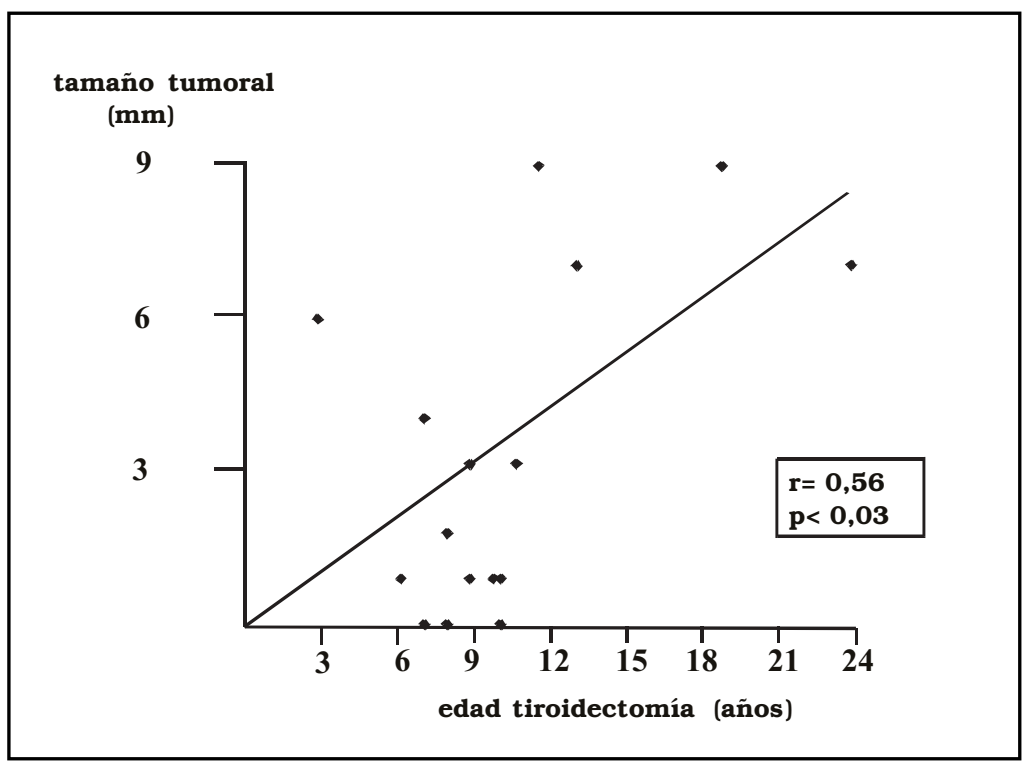

Figura 1. Correlación edad tiroidectomía vs tamaño tumoral. A los sujetos con hiperplasia de células $\mathrm{C}$ se les asignó un tamaño de $0 \mathrm{~mm}$. 


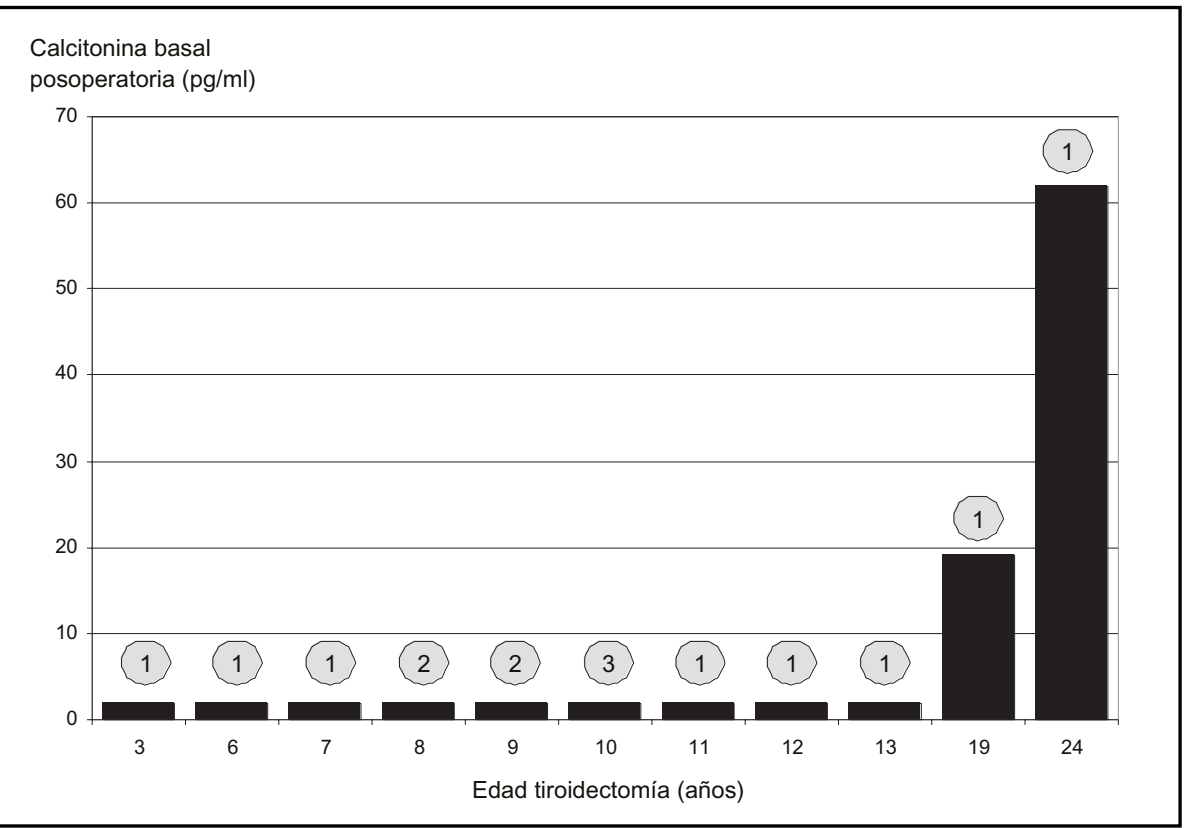

Figura 2. Edad tiroidectomía profiláctica vs calcitonina basal posoperatoria. Los círculos sobre las barras contienen el número de pacientes correspondientes a cada edad. El valor normal de calcitonina basal posoperatoria es $\leq 10 \mathrm{pg} / \mathrm{ml}$.

En todos los pacientes, la ecografía cervical no demostró remanente ni adenopatías. En los dos pacientes no curados bioquímicamente, la búsqueda de metástasis cervicales, mediastínicas, pulmonares, hepáticas u óseas mediante TAC de cuello, mediastino, pulmón y abdomen, así como el cintigrama óseo y cintigrama ${ }^{99 \mathrm{~m}} \mathrm{Tc}-\mathrm{V}-\mathrm{DMSA}$, fue negativa.

Respecto a las complicaciones quirúrgicas, solo un paciente presentó hipoparatiroidismo definitivo a pesar del autotrasplante de células paratiroideas. Los valores actuales de calcio se han mantenido adecuados con uso de calcio y calcitriol. No se observó lesión del recurrente u otra complicación de importancia. El manejo del hipotiroidismo ha sido el problema más frecuente por la falta de adherencia al tratamiento.

El estudio bioquímico en búsqueda de feocromocitoma en todo el período de seguimiento, ha sido negativo en 13 pacientes y no se ha realizado en 3 por razones económicas. La evaluación para la pesquisa de hiperparatiroidismo ha sido negativa y se ha hecho regularmente midiendo calcemia, fosfemia y PTH.

\section{Discusión}

El CMT hereditario se desarrolla a partir de la hiperplasia de las células $C$ del tiroides, el cual es un estado preneoplásico. La transformación histológica desde HCC a CMT es cualitativa, por lo que el uso de criterios cuantitativos sería irrelevante para el diagnóstico ${ }^{3,16}$. Existe un retraso característico en las fases del desarrollo tumoral, desde el estado preneoplásico al CMT, y desde éste al compromiso ganglionar. Esto, sumado al hecho de que el estudio genético permite un diagnóstico preciso, hace del CMT hereditario un excelente modelo para evaluar la utilidad de la terapia intervencionista en pacientes que tienen una predisposición genética para el cáncer ${ }^{16}$. Hay abundante evidencia intemacional de que la TP ofrece la posibilidad de curación incluso antes de que se desarrolle el cáncer medular ${ }^{13,23,24}$. Existe unanimidad respecto a la indicación de realizar TP total, debido al riesgo de multicentricidad que en el CMT hereditario es mucho mayor que en la forma esporádica ( $80 \%$ vs $20 \%)$ y también porque es fundamental erradicar toda la HCC. Este tipo de cirugía ofrece la única posibilidad de cura definitiva ${ }^{1,25}$. 
En nuestro país, el análisis genético del CMT se inició en 1997 y hasta ahora se han reclutado 11 familias con NEM 2. En los 22 casos índices se encontraron mutaciones del proto-oncogen RET, lo que permitió identificar a 25 portadores en riesgo de desarrollar la enfermedad, ninguno de los cuales conocía su condición. Todos los individuos operados e incluidos en este estudio presentaron algún tipo de alteración histológica: 3 con HCC y 13 con CMT, confirmando la confiabilidad del análisis genético y apoyando la idea de que este estudio debe realizarse a temprana edad (Tabla 1) ${ }^{16}$. En nuestros pacientes, la medición de calcitonina basal preoperatoria fue altamente específica (100\%) y sensible (83\%), sin embargo en otras series con mayor número de individuos, estos porcentajes disminuyen ostensiblemente ${ }^{16,18}$. La medición preoperatoria de calcitonina estimulada con pentagastrina sólo se realizó en 7 pacientes. Los niveles no se correlacionaron con el tamaño tumoral-histología: 2 pacientes con HCC mostraron una gran elevación y en un niño de 7 años los valores normales fallaron en predecir el CMT. Históricamente, la prueba de calcitonina estimulada usando pentagastrina se utilizó por décadas para la detección de los portadores asintomáticos de las NEM $2^{7}$; sin embargo, su positividad es dependiente de la edad y además presenta falsos positivos de hasta 15\%, conduciendo a tiroidectomías innecesarias en familiares que no heredaron la mutación $\mathrm{RET}^{13-15}$.

Respecto a la edad en que debiera realizarse la TP, no existe un acuerdo unánime entre los diferentes grupos. El último consenso del taller de NEM (Gubbio, Italia 1999) y otra publicación reciente, recomiendan que los portadores de mutaciones de alto riesgo (codones 611, 618, 620, 634) sean sometidos a tiroidectomía antes de los 5 años de edad ${ }^{17,18}$. El 94\% de nuestros pacientes pertenece a este grupo, y si se consideran las publicaciones antes mencionadas, solo uno de ellos fue operado dentro del plazo sugerido. Este retardo en la cirugía podría explicar el alto porcentaje de CMT observado en nuestro grupo (81\%), cuyos resultados no difieren significativamente de otros estudios con pacientes de edades similares ${ }^{23,26}$. Otros argumentos conocidos a favor de la TP precoz y confirmados por nuestros resultados son: a) a menor edad de la $\mathrm{TP}$, menor tamaño tumoral (Figura 1); b) a mayor edad, menor probabilidad de obtener cura bioquímica, tal como se observó en los sujetos operados a los 19 y 24 años (Figura 2) 27,28. Incluso la edad máxima para la cirugía profiláctica podría ser más baja si consideramos que nuestra paciente de 3 años ya presentaba CMT (Tabla 1), hallazgo que también ha sido reportado en otras publicaciones ${ }^{16}$. Un hecho destacable es que todos nuestros pacientes operados entre los 3 y 13 años se encuentran curados bioquímicamente, aunque estamos conscientes de que se necesita un mayor seguimiento.

En presencia de cualquier CMT esporádico o hereditario, diagnosticado clínicamente, el compromiso ganglionar cervical es $>80 \%$ por lo que hay acuerdo en realizar linfadenectomía central con 0 sin disección ipsilateral o bilateral; sin embargo cuando la cirugía es profiláctica, este aspecto es discutible $^{18,28}$. La presencia de metástasis es excepcional en sujetos $\leq 10$ años con mutaciones del codón 634 (mutación que corresponde casi a 70\% de nuestro estudio), algunos grupos recomiendan la disección ganglionar de rutina a aquellos $>10$ años, aunque la mayoría de los cirujanos realizan disección central independientemente de la edad $^{13,16,17,23,30,31}$. En nuestro estudio no existió un protocolo, pero la conducta general fue la de disecar los ganglios centrales a todos los mayores de 10 años y en el resto de los pacientes, extirpar los ganglios sospechosos a la palpación. Aunque no hubo evidencias de metástasis ganglionares en los 9 sujetos a los cuales se les hizo disección ganglionar, 2 de ellos han persistido con niveles de calcitonina levemente elevados. En estos 2 pacientes, la ecografía cervical (que tiene una sensibilidad de $96 \%$ para detectar metástasis ganglionares) y los otros métodos imagenológicos convencionales y el cintigrama ${ }^{99 \mathrm{~m}} \mathrm{Tc}-\mathrm{V}-\mathrm{DMSA}$ (sensibilidad de $60-68 \%$ para detectar metástasis ganglionares y pulmonares) fueron negativos ${ }^{32}$. La falla en detectar metástasis en nuestros pacientes se podría explicar por las siguientes razones: corto período de seguimiento (5 años promedio), ya que se sabe que hasta $40 \%$ de los pacientes con niveles de calcitonina no detectables e imágenes negativas, evidencian recidiva clínica cuando se siguen por períodos prolongados; falta de disponibilidad de una técnica más sensible para detectar metástasis como la recientemente aparecida tomografía por emisión de positrones con 18-fluorodeoxiglucosa $\left({ }^{18} \mathrm{FDG}\right.$-PET) que 
tiene mayor sensibilidad en la pesquisa de metástasis ganglionares cervicales y mediastínicas o finalmente por causas que aún se desconocen 33,34 .

En conclusión, la tiroidectomía profiláctica en los portadores asintomáticos de las mutaciones del proto-oncogen RET es una cirugía bastante segura y con un porcentaje bajo de complicaciones. Ella debiera realizarse a temprana edad para lograr detectar la enfermedad en etapa preneoplá-

\section{REFERENCIAS}

1. Raue F, Frank-Raue K, Grauer A. Multiple endocrine neoplasia type 2: Clinical features and screening. Endocr Metab Clin North Am 1994; 23: 137-56.

2. Carney JA, Sizemore GW, Hayles aB. Multiple endocrine neoplasia type 2B. Pathobiol Annu 1978; 8: 105-53.

3. Wolfe H, Melvin K, Cervi-Skinner S, SaAdi A, Jular J, Jackson C, Tashjian A. C-cell hyperplasia preceding medullary thyroid carcinoma. N Eng J Med 1973; 289: 437-41.

4. Sipple J. The association of pheochromocytoma with carcinoma of the thyroid gland. Am J Med 1961; 31: 163-6.

5. Tashjian A, Howland B, Meivin K, Hill C. Immunoassay of human calcitonin: clinical measurement, relation to serum calcium and studies in patients with medullary carcinoma. N Engl J Med 1970; 283: 890-5.

6. MeLVIN K, MiLeR H, TASHJian A. Early diagnosis of medullary carcinoma of the thyroid gland by means of calcitonin assay. N Engl J Med 1971; 285: 115-20.

7. Gagel R, Tashian a Jr, Cummings $\mathrm{T}$, Papathanasopoulos N, Kaplan M, Delewis R et al. The clinical outcome of prospective screening for multiple endocrine neoplasia type 2a. N Engl J Med 1988; 318: 478-84.

8. Mathew C, Chin K, Easton D, Thorpe K, Carter C, LiOU G ET AL. A linked genetic marker for multiple endocrine neoplasia type 2A on chromosome 10. Nature 1987; 328: 527-8.

9. Donis-Keuer H, Dou S, Chi D, Carlson, K, Toshima $\mathrm{K}$, Lairmore T et aL. Mutations of the RET proto- sica o de microcarcinoma, evitando el compromiso ganglionar y haciendo más factible la cura bioquímica. Se confirma también que el estudio genético es una herramienta mucho más confiable y precisa que la medición de calcitonina preoperatoria basal o estimulada con pentagastrina, esta última considerada previamente como el estándar de oro en esta patología.

oncogen are associated with MEN 2A and FMTC. Hum Mol Genet 1993; 2: 851-6.

10. Mumgan L, Kwok J, Healey C, Elsdon M, Eng C, GARDNER E ET AL. Germ-line mutations of the RET proto-oncogen in multiple endocrine neoplasia type A. Nature 1993; 363: 458-60.

11. Eng C, Mumigan LM. Mutations of the RET ProtoOncogene in the Multiple Endocrine Neoplasia Type 2 Syndromes, Related Sporadic Tumours and Hirschsprung Disease. Hum Mutat 1997; 9: 97-109.

12. Eng C, Ciayton D, Schuffenecker I, Lenoir G, Cote G, GaGel RF et aL. The relationship between specific RET proto-oncogene mutations and diseases phenotype in multiple endocrine neoplasia type 2A. JAMA 1996; 276: 1575-9.

13. Wells S, Chi D, Toshima K, Dehner L, Coffin S, Dowton B et aL. Predictive DNA testing and prophylactic thyroidectomy in patients at risk for multiple endocrine neoplasia type 2A. Ann Surg 1994; 2203: 237-47.

14. Chi D, Toshima K, Donis-Kelier H, Welis S Jr. Predictive testing for multiple endocrine neoplasia type 2 based on the detection of mutations in the RET proto-oncogene. Surgery 1994; 116: 124-32.

15. Lips CJ, LANDSVATER RM, HOPPENER JW, GeERdink RA, Bljham G, Van Veen JM et al. Clinical screening as compared with DNA analysis in families with multiple endocrine neoplasia type 2A. N Engl J Med 1994; 331: 828-35.

16. Machens A, Niccow-Sire P, Hoegel J, Frank-Raue K, Van VRoonhoven T, Roeher H et al. Early malignant progression of hereditary medullary thyroid cancer. N Engl J Med 2003; 349: 1517-25.

17. Brandi M, Gagel R, Angeli A, Bilezikian J, BeckPeccoz P, Bordi C et al. Guidelines for diagnosis 
and therapy of MEN type 1 and type 2. J Clin Endocrinol Metab 2001; 86: 5658-71.

18. Machens A, UkKat J, BrauckhofF M, Gimm O, Draile $\mathrm{H}$. Advances in the management of hereditary medullary thyroid cancer. J Intern Med 2005; 257: 50-9.

19. Wohwk N, Becker P, Youlton R, Cote G, Gagel R. Mutaciones de línea germinal del proto-oncogen RET en pacientes chilenos con cáncer medular tiroideo hereditario y esporádico. Rev Méd Chile 2001; 129: 713-8.

20. Gagel RF, Cote GJ, Martins Bugalho MJG, Boyd AE, Cummings T, Goepfert $H$ et al. Clinical use of molecular information in the management of multiple endocrine neoplasia type 2A. J Int Med 1995; 238: 333-41.

21. Yip L, Cote GJ, Shapiro SE, Ayers GD, Herzog CE, SeLUN RV ET AL. Multiple endocrine neoplasia type 2: evaluation of the genotype-phenotype relationship. Arch Surg 2003; 138: 409-16.

22. Motté $P$, Vauzeile $P$, Gardel $P$, Ghimani $P$, Cailou $B$, Parmentier $C$ et al. Construction and clinical validation of a sensitive and specific assay for serum mature calcitonin using monoclonal anti-peptide antibodies. Clin Chim Acta 1998; 174: 35-54.

23. Dralie H, Gimm O, Simon D, Frank-Raue K, Gortz G, NiederLe B et al. Prophylactic thyroidectomy in 75 children and adolescents with hereditary medullary thyroid carcinoma: German and Austrian experience. World J Surg 1998; 22: 744-51.

24. Ukkat J, Lorenz K, Hinze R, Thomusch O, Dralie H. Importance of early screening and thyroidectomy in asymptomatic nonindex RET germline carriers. World J Surg 2001; 125: 713-7.

25. Block MA, Jackson CE, TAshjian AH, JR. Management of occult medullary thyroid carcinoma: evidenced only by serum calcitonin level elevations after apparently adequate neck operations. Arch Surg 1978; 113: 368-72.

26. Sanso G, Domene H, García Rudaz M, Pusiol E, De Mondino A, Roque M ET AL. Very early detection of
RET proto-oncogen mutation is crucial for preventive thyroidectomy in multiple endocrine neoplasia type 2 children. Cancer 2002; 94: 323-30.

27. Cohen R, Campos JM, Salaun C, Heshmati HM, Kraimps JL, Proye C et al. Preoperative calcitonin levels are predictive of tumor size and postoperative calcitonin normalization in medullary thyroid carcinoma. Groupe d'etudes des tumeurs a calcitonine (GETC). J Clin Endocrinol Metab 2000; 85: 919-22.

28. Modiglani E, Cohen R, Campos JM, Conte-Devolx B, Maes B, Boneu A et al. Prognostic factors for survival and for biochemical cure in medullary thyroid carcinoma: results in 899 patients. Groupe d'etude des tumeurs a calcitonine. Clin Endocrinol 1998; 48: 265-73.

29. Scolo C, Baudin E, Travagli JP, Cailou B, Bellon N, LeBouleuX S ET al. Rationale for central and bilateral lymph node dissection in sporadic and hereditary medullary thyroid cancer. J Clin Endocrinol Metab 2003; 88: 2070-5.

30. Evans DB, Fieming JB, Lee JE, Cote GJ, Gagel RF. The surgical treatment of medullary thyroid carcinoma. Semin Surg Oncol 1999; 16: 50-63.

31. CoHen M, Moley J. Surgical treatment of medullary thyroid carcinoma. Journal of Internal Medicine 2003; 253: 616-26.

32. Brandt-Mainz K, Muler SP, Gorges R, Sauer B, Bоскіsсн A. The value of fluorine-18 fluorodeoxyglucose PET in patients with medullary thyroid cancer. Eur J Nucl Med 2000; 27: 490-6.

33. Peluegriti G, Lebouleux S, Baudin E, Belon N, Scolo C, Travagl JP et al. Long-term outcome of medullary thyroid carcinoma in patients with normal postoperative medical imaging. Br J Cancer 2003; 88: 1537-42.

34. Szakall S Jr, Esik O, Bajzik G, Repa I, Dabasi G, SINKOVICS I ET AL. 18F-FDG PET detection of lymph node metastases in medullary thyroid carcinoma. J Nucl Med 2002; 43: 66-71.

Agradecimientos

A los doctores Angélica Montecinos, Teresa Vidal, Roberto Wiener, Eugenio Arteaga y socios de la SOCHEM por permitimos estudiar sus pacientes. A los doctores Aníbal Baeza y Carlos Ferrada por el excelente manejo quirúrgico de los pacientes que tuvieron a su cargo. 